\title{
A propos des apports nutritionnels conseillés (ANC) en lipides
}

Oléagineux, Corps Gras, Lipides. Volume 8, Numéro 1, 13-4, Janvier - Février 2001, La filière

Auteur(s) : Philippe LEGRAND, Georges DURAND, Laboratoire de biochimie, École nationale supérieure agronomique de Rennes, Institut national de la recherche agronomique, 65 , rue de Saint-Brieuc - CS 84215, 35042 Rennes Cedex, France.

Résumé : Les apports nutritionnels conseillés (ANC) qui viennent d'être publiés sont le fruit d'un long travail collectif ayant impliqué près de cent auteurs [1]. En ce qui concerne les lipides, la commission était composée de cinq personnes : J.-M. Bourre, B. Descomps, G. Durand, S. Renaud et P. Legrand. Par rapport à l'édition antérieure (1992), il a été demandé de traiter non seulement les acides gras essentiels mais également les acides gras saturés, mono-insaturés, le cholestérol, etc., et la part des lipides dans l'apport calorique. En complément, une approche par groupe d'âge et/ou statut physiologique est également présentée (nouveau-né, femme enceinte ou allaitante, personne âgée). Sans être révolutionnaires, ces nouveaux ANC proposent plusieurs grandes orientations dictées par l'état des connaissances scientifiques.

Mots-clés : lipides, apports conseillés, nutrition.

Summary : The french nutritional recommendations have been recently published. The "lipids" chapter is discussed here. Emphasis is given to the prevention aspects which lead to point out the importance of the linoleic/alpha-linolenic acid ratio.

Keywords : lipids, recommendations, nutrition

\section{ARTICLE}

Tout d'abord a été proposé l'intervalle de 30-35\% d'énergie d'origine lipidique. Le manque de données convaincantes sur le sujet invite à tenir cette position mesurée par rapport à la tendance d'autres comités étrangers qui veulent réduire plus encore cette part des lipides. En effet, en deçà de $30 \%$, l'apport équilibré en acides gras essentiels est difficile à réaliser, compte tenu de la composition des aliments usuels.

En ce qui concerne les apports en acides gras chez l'adulte, il convient, pour commenter et " adoucir " la sécheresse d'un tableau de chiffres, d'expliquer la démarche employée, avec ses deux aspects les plus importants.

* Les ANC n'ont pas la même force pour tous les acides gras. Les acides gras poly-insaturés font l'objet de nombreuses études et leur apport est celui de nutriments bien connus comme indispensables à la fonction de nutrition de l'organisme. Les acides gras saturés sont eux historiquement liés à des risques cardiovasculaires en cas de consommation excessive et sont donc 
abordés dans une logique de limite à considérer, mais sans caractère trop strict. Pour les acides gras mono-insaturés enfin, c'est-à-dire, dans la pratique, l'acide oléique, c'est ici la considération que sa neutralité est son principal avantage qui justifie de compléter l'apport lipidique avec lui, une fois les poly-insaturés satisfaits et les saturés limités.

* La seconde considération est que la fonction de prévention des maladies a pris sans cesse de l'importance et ne peut plus être vraiment séparée de la fonction de nutrition, puisque les déficiences en acides gras n'existent apparemment pas dans nos pays. C'est pourquoi les paragraphes concernant les " maladies cardiovasculaires » et le " cancer » ont pris de l'importance par rapport aux éditions précédentes, importance nourrie par une très abondante littérature, en particulier dans le domaine du cancer.

Ainsi introduites, les grandes orientations sont les suivantes.

L'apport en acide linoléique est précisé à $4 \%$ du contenu énergétique et l'apport en acide alphalinolénique est précisé à $0,8 \%$ du contenu énergétique. Ce resserrement des chiffres par rapport à l'édition précédente s'explique, au vu de la bibliographie récente, par le risque de surcharge en acide linoléique et la nécessité de maintenir un apport suffisant en acide alpha-linolénique. Le rapport linoléique/alpha-linolénique devrait tendre vers la valeur de 5 , ce qui est loin d'être le cas dans la population française.

Cette évolution correspond à une mise au point bibliographique nécessaire, suite au trop fort effet de balancier qui avait, dans le passé, condamné vigoureusement les acides gras saturés et donné aux n-6 des vertus exagérées, en sous-estimant la nécessité des $n-3$. L'édition précédente indiquait d'ailleurs déjà : " un régime riche en acide linoléique n'a pas eu sur la mortalité coronarienne les effets protecteurs escomptés ". La position des nouveaux ANC est en fait très mesurée, si on la compare à celle de certains comités étrangers qui envisagent des valeurs du rapport linoléique/alpha-linolénique de 2 ou 3.

Concernant les acides gras à très longues chaînes, une valeur est proposée sans distinction des deux familles $n-6$ et $n-3$ (faute de données suffisantes), avec néanmoins une précision concernant le DHA (acide docosahexaénoïque, 22:6n-3) dont l'importance est démontrée dans la bibliographie récente.

Les paragraphes " maladies cardiovasculaires " et " cancer " appellent peut-être un commentaire particulier : il n'est bien sûr pas question de toxicité, ni de caractère cancérigène pour des molécules qui sont des nutriments indispensables, mais il est utile de souligner que les connaissances scientifiques montrent depuis longtemps que, dans le cadre de la prévention de ces maladies, l'ensemble de ces molécules est requis à des doses précisément équilibrées entre elles, du fait de leurs rôles souvent antagonistes dans des fonctions extrêmement importantes comme, par exemple, l'agrégation plaquettaire, l'inflammation, etc.

Le passé a, hélas, été riche en simplifications abusives, en descriptions caricaturales du bon et du mauvais, au prétexte que le consommateur ne peut saisir que des arguments simples. Présenter dans les ANC la réalité complexe des rôles des différents acides gras, en soulignant leurs interactions (compétitions pour les désaturases, pour la cyclo-oxygénase, pour la lipoxygénase, etc.), ne doit pas conduire à des fausses interprétations dues à une lecture incomplète ou à une mauvaise compréhension. 
Cette réalité complexe doit plutôt inciter l'utilisateur des ANC à mieux s'informer. Les ANC constituent donc aussi un vecteur d'éducation nutritionnelle.

Parmi les autres points nouvellement exprimés, le cas des personnes âgées a été abordé pour constater finalement que leurs besoins ne sont guère spécifiques en tenant néanmoins compte d'un apport énergétique total plus faible.

Concernant le nouveau-né, les connaissances sont maintenant mieux établies et permettent de recommander la supplémentation des laits infantiles en acides gras poly-insaturés à très longue chaîne, pour se rapprocher de la composition du lait de femme. Il faut souligner de plus que, dans le cas du nouveau-né, à la différence de l'adulte, le rapport linoléique/alpha-linolénique doit cette fois être compris entre 5 et 10 .

Enfin le paragraphe " cholestérol " montre l'inutilité de réduire le cholestérol alimentaire dans la population générale non hypercholestérolémique.

Les ANC constituent un point bibliographique allant des études épidémiologiques et des études physiologiques aux études in vitro sur lignées cellulaires. Des controverses demeurent, et c'est pourquoi tout n'est pas traité, tout n'est pas affirmé avec la même force. Des études sont en cours, d'autres sont nécessaires, en particulier dans le domaine des fonctions des acides gras saturés, des acides gras conjugués et dans le domaine des apports en acide oléique. Ambroise Martin (coordonnateur général) avait fait appel dans les colonnes de la presse spécialisée à tous les avis et contributions sur les ANC. Ils furent souvent très utiles et appréciés.

Les ANC sont-ils facilement réalisables compte tenu de la composition des aliments disponibles ? Disons que pour aider le consommateur qui le souhaite à tendre vers les ANC, certains produits et certaines compositions lipidiques doivent évoluer, certains usages doivent se modifier, des produits nouveaux peuvent émerger, mais des pratiques publicitaires doivent aussi évoluer. Les industriels, très généralement compétents et à la pointe de la bibliographie, feront sûrement bon usage des nouveaux ANC. Il est indispensable aussi que les éducateurs, les praticiens (médecins, diététiciens) et surtout les journalistes fassent l'indispensable démarche d'un minimum de formation pour participer à l'éducation nutritionnelle du consommateur qui, elle, constitue une véritable carence.

\section{REFERENCES}

1. Apports nutritionnels conseillés pour la population française. Paris : Afssa, éd. Tec \& Doc, 2000. 